\title{
Análisis psicométrico de una escala de dependencia emocional en universitarios peruanos
}

\section{Psychometric Analysis of a Scale of Emotional Dependence in Peruvian College Students}

\author{
José Ventura ${ }^{\mathrm{a}} \&$ Tomás Caycho ${ }^{\mathrm{b}}$ \\ ${ }^{a}$ Universidad de San Martín de Porres, Lima, Perú \\ ${ }^{b}$ Universidad Privada del Norte, Lima, Perú
}

\begin{abstract}
Resumen: El presente artículo analiza las propiedades psicométricas de la Escala de Dependencia Emocional, de Lemos Hoyos y Londoño Arredondo (2006), compuesta por 23 ítems, con seis alternativas de respuesta tipo Likert. Los participantes fueron 520 universitarios peruanos (147 varones y 373 mujeres), cuyas edades fluctuaron entre los 16 a 47 años, con un promedio de 22,28. Los participantes fueron divididos en una muestra de 200 personas para el análisis factorial exploratorio, y 320 , para el análisis factorial confirmatorio. El análisis de correlación ítem-test indicó una adecuada homogeneidad de los ítems. La confiabilidad fue analizada mediante el coeficiente omega, variando de acuerdo a los modelos, desde ,85 a ,93. El análisis factorial exploratorio sugirió la existencia de un solo factor. Debido a la diferencia con la versión original, se realizó el análisis factorial confirmatorio, concluyendo que el modelo de un factor general con seis factores específicos tiene aceptables valores de bondad de ajuste, pero no cuenta con valores de fiabilidad buenos para la muestra en estudio.
\end{abstract}

Palabras clave: dependencia emocional, confiabilidad, análisis factorial.
Abstract: The present article analyzes the psychometric properties of the Emotional Dependence Scale, developed by Lemos Hoyos and Londoño Arredondo (2006), composed by 23 items, with six Likert type response alternatives. A total of 520 students ( 147 males and 373 females) participated in the study, ranging in age from 16 to 47 years old, with an average of 22.28 years. The participants were divided into a sample of 200 people for the exploratory factor analysis and 320, for confirmatory factor analysis. The analysis of the item-test correlation indicated a suitable homogeneity of the items. The reliability was analyzed by means of the coefficient omega, varying according to the models, from .85 to .93 . Exploratory factor analysis suggested the existence of a single factor. Due to the difference with the original version, confirmatory factor analysis was performed, concluding that a general factor model with six specific factors has acceptable values of goodness of fit, but does not have good reliability values for the sample under study.

Keywords: emotional dependency, reliability, factor analysis.

Los autores agradecen a la Facultad de Ciencias de la Comunicación, Turismo y Psicología, de la Universidad de San Martín de Porres, y a la Facultad de Ciencias de la Salud, de la Universidad Privada del Norte, por el apoyo brindado durante la realización del estudio reportado.

Contacto: J. Ventura, Facultad de Ciencias de la Comunicación, Turismo y Psicología de la Universidad de San Martín de Porres, Av. Tomás Marsano 242, Lima 34. Correo electrónico: jventuraleon@ gmail.com

Cómo citar: Ventura, J. \& Caycho, T. (2016). Análisis psicométrico de una escala de dependencia emocional en universitarios peruanos. Revista de Psicología, 25(1), 1-17.

http://dx.doi.org/10.5354/0719-0581.2016.42453 


\section{Introducción}

A partir de la década de 1980 y 1990, el concepto de dependencia emocional se popularizó gracias a la aparición de numerosos libros de divulgación (Bireda, 1998; Mellody, 2006; Norwood, 1985; Riso, 1999). Esta literatura ha aportado información al público general, haciendo visible la problemática; no obstante, son documentos basados más en la experiencia profesional de los autores que en evidencia empírica.

Sirvent y Moral (2007), plantearon el concepto de dependencia relacional, para referirse a un conjunto de dependencias suscitadas en el ámbito de las relaciones interpersonales. En su clasificación, los autores dividen las dependencias relacionales en: a) genuinas, donde se encuentra la dependencia emocional, la adicción al amor, el trastorno de personalidad por dependencia y el apego ansioso, y; b) mediatizadas, donde aparecen la adicción a drogas, la codependencia y la bidependencia.

Por otro lado, Castelló Blasco define la dependencia emocional como "una necesidad extrema de carácter afectivo que una persona siente hacia su pareja a lo largo de diferentes relaciones" (2005, p. 17). Esta definición ha sido utilizada en diversas investigaciones sobre dependencia emocional (Aiquipa T., 2012; Lemos Hoyos \& Londoño Arredondo, 2006). El mismo Castelló Blasco (2005), señala que las características de una persona con dependencia emocional son: necesidad excesiva del otro (deseo de acceso constante hacia él o ella), deseos de exclusividad en la relación, prioridad de la pareja sobre cualquier cosa, idealización del objeto, relaciones basadas en la sumisión y la subordinación, historia de relaciones de pareja desequilibradas, miedo a la rup- tura y, finalmente, asunción del sistema de creencias de la pareja.

El estudio de la dependencia emocional es importante en su relación con otras variables psicosociales. Así, existe una relación negativa entre la sobredependencia destructiva y la masculinidad; mientras que la dependencia saludable se encuentra correlacionada de manera positiva con la masculinidad y feminidad (Bornstein et al., 2004). De la misma manera, se puede observar que la dependencia emocional se encuentra asociada con la falta de confianza en sí mismo/a, ansiedad de separación, expresión afectiva, búsqueda de atención, depresión ante las pérdidas, mala calidad de la relación conyugal, distorsiones cognitivas, intentos de suicidio y violencia de pareja (Aiquipa Tello, 2015; Bornstein \& O'Neill, 2000; Hirschfeld et al., 1977; Lemos Hoyos \& Londoño Arredondo, 2006; Lemos Hoyos, Londoño Arredondo, \& Zapata Echavarría, 2007; Sanathara, Gardner, Prescott, \& Kendler, 2003).

La evaluación de la dependencia emocional es un aspecto importante, contando en la actualidad con diversos instrumentos destinados a su medición. Así, se puede mencionar la existencia de la Escala Específica de Dependencia Conyugal (Spouse Specific Dependency Scale), desarrollada por Rathus y O'Leary (1997), y del Inventario de Dependencia Interpersonal (Interpersonal Dependency Inventory), de Hirschfeld et al. (1977), que parte del concepto de dependencia interpersonal como un conjunto de pensamientos, sentimientos y comportamientos asociados a una necesidad de cercanía con otras personas. Es posible mencionar también el Test de Perfil Relacional (Relational Profile Test), de Bornstein, Geiselman, Eisenhart y Languirand (2002), 
que mide la dependencia a partir de tres dimensiones asociadas: sobredependencia destructiva, desapego disfuncional y dependencia saludable. Más actual es el Test de Dependencias Sentimentales, de Sirvent y Moral (2005). Mientras que, en el Perú, se ha desarrollado el Inventario de Dependencia Emocional (Aiquipa T., 2012), que explica el constructo en relación a siete factores: Miedo a la ruptura, Miedo e intolerancia a la soledad, Prioridad de la pareja, Necesidad de acceso a la pareja, Deseos de exclusividad, Subordinación y sumisión, Deseos de control y dominio.

Dentro de los diferentes instrumentos de evaluación de la dependencia emocional, el Cuestionario de Dependencia Emocional (CDE) de Lemos Hoyos y Londoño Arredondo (2006), ha sido empleado en diversas investigaciones (Jaller Jaramillo \& Lemos Hoyos, 2009; Lemos Hoyos, Jaller Jaramillo, González Calle, Díaz León, \& De la Ossa, 2012; Lemos Hoyos et al., 2007). Originalmente, Lemos Hoyos y Londoño Arredondo (2006) reportaron información psicométrica del CDE en 815 participantes de Medellín (Colombia), obteniéndose un alfa de Cronbach de ,93 para la escala total. El análisis factorial exploratorio permitió observar la existencia de seis factores o dimensiones: Ansiedad de separación $(\alpha=, 87)$, Expresión afectiva de la pareja $(\alpha=, 84)$, Modificación de planes $(\alpha=, 75)$, Miedo a la soledad $(\alpha=, 80)$, Expresión límite $(\alpha=$ ,62) y Búsqueda de atención $(\alpha=, 78)$.

A pesar de que el CDE es utilizado en diversos estudios (González-Jiménez \& Hernández-Romera, 2014; Lemos Hoyos et al., 2007; 2012), estos se han basado en las propiedades psicométricas originales, sin realizar análisis psicométricos de la escala de acuerdo a sus muestras de estudios. Se ha reportado un solo estudio psicométrico, de Méndez Zavala, Favila Fi- gueroa, Valencia Cruz y Díaz Loving (2012), quienes estudiaron la estructura psicométrica del CDE en una muestra de 93 individuos mexicanos, entre los 16 y 55 años de edad. Se utilizó el índice de discriminación de los reactivos de los puntajes extremos mediante la prueba $t$ de Student, evidenciándose que todos los reactivos direccionan de manera adecuada. El análisis de la estructura interna se realizó mediante el método de componentes principales con rotación ortogonal (Varimax), previa a ello, se comprobaron las medidas de adecuación muestral, como la prueba de esfericidad de Bartlett, que resultó significativa, y el valor de la prueba Kaiser-Meyer-Olkin, que fue de ,85. El análisis factorial reportó siete factores, no obstante, aquel componente con valor menor a 1 ; reactivos con carga factorial menor a ,40 o que no estuviera cargando en un solo factor, fueron eliminados, quedando al final solo dos factores, denominados Ansiedad por separación y Expresiones límite, que en conjunto explican $62,58 \%$ de la varianza. Se exploró la confiabilidad por medio de alfa de Cronbach para el total de la prueba, dando como resultado ,90. Además, de un alfa por cada componente $(\alpha$ Ansiedad por separación $=, 88 ; \alpha$ Expresión limite $=, 60)$.

En el contexto peruano, no existen estudios de validación del CDE, además de un bajo número de investigaciones publicadas en relación a la dependencia emocional. Si bien existe el Inventario de Dependencia Emocional (Aiquipa T., 2012), este solo cuenta con estudios de validez mediante análisis factorial exploratorio, método que presenta una serie de deficiencias, que son corregidas mediante el empleo del análisis confirmatorio (Batista-Foguet, Coenders, \& Alonso, 2004). El análisis confirmatorio permite delimitar el concepto de factor común como aquel subyacente a indicadores concretos, evi- 
tando introducir factores de difícil interpretación. De igual manera, permite realizar contrastes estadísticos de las hipótesis especificadas. Por otro lado, el análisis confirmatorio no asume igualdad de las saturaciones ni de las varianzas de error, lo que permite someter estos supuestos a contrastes estadísticos, lo que estaría en relación con la evaluación de la fiabilidad (Batista-Foguet et al., 2004).

La falta de estudios de validación del CDE en el Perú y otros países, justifica la adaptación de los instrumentos de medición psicológica a las diferentes culturas y variantes idiomáticas (Muñiz \& Hambleton, 1996). En psicología los test son altamente dependientes de los aspectos culturales, razón por la cual, se debe verificar si el constructo a evaluar es comparable con la cultura de procedencia del test (Fernández, Pérez, Alderete, Richaud, \& Fernández Liporace, 2010).

En base a la información mencionada, esta investigación tiene como propósito realizar el análisis psicométrico del CDE en una muestra de estudiantes universitarios peruanos. La revisión psicométrica del CDE es importante, ya que tiene implicancias tanto teóricas como prácticas. Desde el punto de vista teórico, ayudará a la revisión del constructo de dependencia emocional y las dimensiones que plantean originalmente Lemos Hoyos y Londoño Arredondo (2006). Desde el punto de vista práctico, permitirá que el cuestionario pueda ser empleado en el ámbito clínico, con mayor validez y confiabilidad, para el diagnóstico de personas con dependencia emocional.

\section{Método}

La presente investigación es de tipo instrumental, debido a que responde a problemas orientados a demostrar las propie- dades psicométricas de los instrumentos de medición (Montero \& León, 2007).

\section{Participantes}

El CDE se aplicó a 520 estudiantes, pertenecientes a dos universidades de Lima Metropolitana, 147 varones y 373 mujeres, cuyas edades oscilaron entre los 16 a 47 años, cuyo promedio fue 22,28 y con una desviación estándar de 5,62. Los participantes pertenecían al nivel socioeconómico medio, seleccionado mediante un método no probabilístico de tipo intencional (Hernández Sampieri, Fernández Collado, \& Baptista Lucio, 2014). La muestra fue dividida en dos, mediante un muestreo aleatorio producido por el programa estadístico utilizado, siguiendo la recomendación de Harrington (2008), que indica que el Análisis Factorial Confirmatorio (AFC) debe ser trabajado en una nueva muestra para confirmar la estructura identificada en el Análisis Factorial Exploratorio (AFE).

La muestra de 200 personas para el AFE estuvo conformada por 43 varones y 157 mujeres, con un promedio de 22,96 años y una desviación estándar de 5,23. La determinación de la cantidad de participantes fue en base a la recomendación de Morales Vallejo (2013), quien sugiere que, para este tipo de análisis, el número mínimo de personas es de 200, o de 5 participantes por cada ítem. Por otro lado, la muestra para el AFC estuvo conformada por 320 personas, 216 mujeres y 104 varones, con un promedio de 21,86 años de edad y una desviación estándar de 5,81.

\section{Instrumento}

Como instrumento de investigación para este estudio, se utilizó el CDE (Lemos Hoyos \& Londoño Arredondo, 2006), el cual contiene 23 ítems. Su alternativa de res- 
puesta es de tipo Likert del 1 al 6, siendo:

1) Completamente falso de mí; 2) El mayor parte falso de mí; 3) Ligeramente más verdadero que falso; 4) Moderadamente verdadero de mí; 5) El mayor parte verdadero de mí, y; 6) Me describe perfectamente.

En relación a sus propiedades psicométricas, el CDE presenta un alfa de Cronbach de ,93; mientras que los seis factores, encontrados mediante análisis factorial exploratorio, explican el $64,7 \%$ de la varianza total. El CDE está compuesto por las siguientes seis dimensiones: Factor 1: Ansiedad de separación, que evalúa las expresiones de miedo que siente una persona ante la posibilidad de disolverse la relación (compuesto por siete ítems); Factor 2: Expresión afectiva de la pareja, que evalúa la necesidad de recibir frecuentes expresiones de afecto, que reafirman el amor que siente, calmando la sensación de inseguridad (compuesto por cuatro ítems); Factor 3: Modificación de planes, mide cambio de actividades, planes y comportamientos, que buscan satisfacer a la pareja o la simple posibilidad de compartir mayor tiempo con él/ella. En el largo plazo, genera aislamiento de las amistades $\mathrm{u}$ otras actividades (compuesto por cuatro ítems); Factor 4: Miedo a la soledad, evalúa temor por permanecer en soledad, por no tener una pareja, no sentirse amado; situación que la persona evita, acercándose más a su pareja y convirtiéndolo/a en una fuente de seguridad y equilibrio (integrado por tres ítems); Factor 5: Expresión límite, mide acciones impulsivas de autoagresión que evitan que la relación termine, porque si aquello sucede, tendrá que enfrentarse a la soledad y sentirá que su vida no tiene sentido (compuesto por tres ítems); Factor 6: Búsqueda de atención, mide esfuerzos activos para obtener la atención de la pareja y asegurar su permanencia en la rela- ción, tratando de ser el centro en su vida (está integrado por dos ítems).

\section{Procedimientos}

Inicialmente, se aplicó el CDE a un grupo piloto de 30 personas, con el fin de comprobar la claridad de los ítems. Los participantes respondieron a las preguntas " $i$ se entiende?" y "¿es claro?”, con lo cual se modificaron algunos ítems que los participantes indicaron no comprender a qué se referían.

Acto seguido, la escala fue revisada por cinco jueces especialistas, tres de ellos expertos en el constructo, y dos expertos en psicometría, quienes establecieron, mediante un formato de criterio de jueces, qué términos de los ítems no presentaban claridad, para los cuales dieron recomendaciones.

Luego de este proceso, se administró el CDE a los estudiantes universitarios dentro de sus aulas, informándoseles del objetivo de la investigación y de las condiciones de anonimato. Cada examinador, previamente entrenado en la aplicación del cuestionario, leyó las instrucciones de este a los participantes y respondieron a sus dudas. Por otro lado, una parte de los cuestionarios fue recolectada de forma virtual, cuyo enlace fue compartido por redes sociales.

En ambas condiciones de recolección de datos, los participantes resolvieron el cuestionario voluntaria y anónimamente, dando su conformidad mediante una ficha de consentimiento informado, que garantiza la confidencialidad de los datos suministrados. Una vez aplicado el instrumento, se excluyeron del análisis aquellos participantes que omitieron respuestas. 


\section{Análisis de datos}

Se utilizó el programa estadístico FACTOR versión 9.2 (Universitat Rovira i Virigili, 2013), para el análisis factorial exploratorio, y el programa $\mathrm{R}$ versión 3.1.2 (R Development Core Team, 2007) para los cálculos de confiabilidad, correlación ítem-test corregida y análisis factorial confirmatorio. Cabe mencionar que ambos programas estadísticos son de libre acceso.

En la primera etapa del análisis estadístico, se analizó la distribución de los ítems, examinando los coeficientes de curtosis y asimetría (Hair, Anderson, Tatham, \& Black, 2004). Asimismo, se examinó la multicolinealidad entre los ítems (Kline, 2011) y se revisó la correlación ítem-test de cada uno de los ítems de la escala total, siguiendo el criterio de Kline (1986), de retirar ítems con valores menores a ,20.

En la segunda etapa, se realizó el análisis factorial exploratorio, con el fin de identificar la estructura que subyace a los ítems (Tabachnick \& Fidell, 2007; Thompson, 2004) con una muestra de 200 participantes. Se estimó la matriz de correlaciones policóricas a los ítems del cuestionario (Freiberg Hoffman, Stover, de la Iglesia, \& Fernández Liporace, 2013). Con los datos resultantes, se examinó si eran factorizables a través del coeficiente de KaiserMeyer-Olkin (KMO; Kaiser, 1970), que permite comparar si las correlaciones entre los ítems son tan altas como para demostrar la existencia de factores comunes; valores cercanos a ,80 sugieren que las correlaciones entre los ítems pueden ser explicadas por otras variables (Kaiser, 1974).

Asimismo, se realizó la prueba de esfericidad de Bartlett (Bartlett, 1950), que permite aceptar o rechazar la hipótesis nula de que la matriz de correlaciones es igual a la matriz de identidad, siendo las correlaciones entre los ítems iguales a cero.

Para la determinación del número de factores, se utilizó el método de análisis paralelo basado en el análisis factorial de rango mínimo (Timmerman \& LorenzoSeva, 2011). La extracción de factores se realizó por medio del método mínimos cuadrados no ponderados, debido a que las variables no se distribuyen de forma normal (Costello \& Osborne, 2005) y el método de rotación fue Promin (LorenzoSeva, 1999).

En la tercera etapa del análisis estadístico, se calculó la fiabilidad mediante el coeficiente omega (McDonald, 1999), y no mediante el coeficiente alfa de Cronbach, debido a que permite analizar el grado de consistencia interna en base a las cargas factoriales y no depende del número de ítems, como sí lo hace el coeficiente alfa, algo oportuno, en vista a que los factores Búsqueda de atención y Miedo a la soledad tienen dos y tres ítems respectivamente. Adicionalmente, se calculó la varianza extraída promedio (AVE, por su nombre en inglés Average Variance Extracted), que indica que la varianza extraída por el factor es más grande que la debida al error de medida, valores mayores a ,50 son reflejo de un buen modelo (Fornell \& Larcker, 1981).

En la cuarta etapa, se verificó la estructura factorial mediante el $\mathrm{AFC}$, con una muestra diferente al AFE de 320 participantes. Para tales fines, se calcularon los índices de bondad de ajuste comparativo y se utilizaron métodos robustos para evaluar el ajuste del modelo. De este modo, se optó por usar la corrección para datos no-normales, método de estimación robusta de Satorra-Bentler (S-B; Satorra \& Bentler, 2001). Se estimó el índice de ajuste no normado (NNFI, por su nombre 
en inglés Not Normed Fit Index) y el índice de ajuste comparativo (CFI, por su nombre en inglés Comparative Fit Index). Adicionalmente, se consideró el error cuadrático medio de aproximación (RMSEA, por su nombre en inglés Root Mean Square Error of Approximation), la raíz residual estandarizada cuadrática media (SRMSR, por su nombre en inglés Standardized Root Mean Square Residual) y el criterio de información de Akaike (AIC, por su nombre en inglés Akaike Information Criterion).

Finalmente, en vista a que se probaron modelos bifactor, se realizó el cálculo de índices de resistencia como varianza común explicada (ECV, por su nombre en inglés Explained Common Variance) y porcentaje de correlaciones no contaminadas (PUC, por su nombre en inglés Percentage of uncontaminated correlations) (Ríos \& Wells, 2014).

\section{Resultados}

\section{Análisis preliminar de los ítems}

Inicialmente, se efectuó el análisis descriptivo de los ítems, examinando la media, desviación estándar, asimetría y curtosis (ver tabla 1). Se observó que los ítems presentaron valores superiores +/- 1,5 (Ferrando \& Anguiano-Carrasco, 2010; Pérez \& Medrano, 2010), concluyendo la presencia de no-normalidad multivariada. Asimismo, la tabla 1 permite observar la correlación ítem-test para cada uno de los ítems, los cuales son superiores al valor ,20 propuesto por Kline (1986), oscilando entre ,35 a ,69. El ítem 15 es el que presenta un mayor valor de correlación ítem-test, dentro de todos los ítems. Asimismo, se examinó la multicolinealidad entre los ítems (Kline, 2011) para descartar variables redundantes; es decir, correlaciones inter-ítem mayores de, 95 .
Tabla 1

Análisis descriptivos para cada uno de los items $(\mathrm{N}=520)$

\begin{tabular}{ccccccc}
\hline Ítem & $M$ & $D E$ & Asimetría & \multicolumn{2}{c}{ Curtosis $\mathrm{r}_{\text {itc }}$} \\
\hline 1 & 1,89 & 1,32 & 1,38 & 1,44 &, 35 \\
2 & 2,19 & 1,63 & 0,94 & 0,05 &, 58 \\
3 & 3,57 & 2,08 & $-0,11$ & $-0,80$ &, 35 \\
4 & 2,83 & 2,13 & 0,45 & $-0,74$ &, 47 \\
5 & 3,29 & 1,88 & 0,13 & $-0,71$ &, 54 \\
6 & 2,38 & 1,89 & 0,85 & $-0,14$ &, 55 \\
7 & 2,54 & 1,85 & 0,66 & $-0,41$ &, 59 \\
8 & 2,37 & 1,71 & 0,86 & 0,02 &, 60 \\
9 & 1,43 & 1,09 & 2,79 & 7,44 &, 40 \\
10 & 1,89 & 1,49 & 1,46 & 1,58 &, 49 \\
11 & 2,79 & 1,77 & 0,49 & $-0,43$ &, 63 \\
12 & 2,97 & 2,30 & 0,42 & $-0,83$ &, 39 \\
13 & 2,28 & 1,62 & 0,93 & 0,27 &, 62 \\
14 & 2,61 & 1,63 & 0,58 & $-0,17$ &, 62 \\
15 & 2,05 & 1,59 & 1,23 & 0,95 &, 69 \\
16 & 2,60 & 1,95 & 0,71 & $-0,26$ &, 52 \\
17 & 2,56 & 1,95 & 0,71 & $-0,26$ &, 54 \\
18 & 1,92 & 1,35 & 1,47 & 2,03 &, 59 \\
19 & 2,37 & 2,08 & 0,92 & $-0,06$ &, 41 \\
20 & 1,71 & 1,38 & 1,83 & 2,87 &, 50 \\
21 & 2,44 & 1,67 & 0,95 & 0,46 &, 48 \\
22 & 2,44 & 1,91 & 0,77 & $-0,25$ &, 46 \\
23 & 2,00 & 1,77 & 1,38 & 1,22 &, 45 \\
\hline
\end{tabular}

Nota: $M=$ Media; $D E=$ Desviación Estándar; $r_{i t c}=$ Correlación ítem-test corregida.

El análisis de la multicolinealidad entre los ítems, evidenció que ninguna correlación en la matriz inter-ítem fue superior a ,90; por lo que no se descartaron ítems mediante este paso.

Análisis factorial exploratorio. Se calcularon los índices de adecuación muestral, observando que los niveles de factorización fueron considerados como buenos $\left(\mathrm{KMO}=, 87, \chi^{2}=2146,5\right.$; Bartlett, $g l=253, p<, 05)$, lo cual indica que es apropiado realizar el análisis factorial en 
la presente muestra. Para la determinación del número de factores, se realizó el análisis paralelo, que sugirió la existencia de que un solo factor subyace a los ítems; así mismo, el método de estimación fue mínimos cuadrados no ponderado con rota- ción promin (Lorenzo-Seva, 1999), elegido debido a la distribución no paramétrica de los datos (Costello \& Osborne, 2005). En la tabla 2, se reporta lo antes mencionado.

Tabla 2

Matriz patrón: análisis factorial exploratorio con un factor del CDE $(\mathrm{N}=200)$

\begin{tabular}{lrr}
\hline Ítems & FG & $\mathrm{h}^{2}$ \\
1. Me siento desprotegido/a cuando estoy solo/a. &, 53 &, 28 \\
2. Me preocupa la idea de ser abandonado/a por mi pareja. &, 67 &, 44 \\
3. Para atraer a mi pareja busco impresionarla/o o divertirla/o. &, 37 &, 14 \\
4. Hago todo lo posible por ser el centro de atención en la vida de mi pareja. &, 57 &, 33 \\
5. Necesito constantemente expresiones de afecto de mi pareja. &, 59 &, 35 \\
6. Si mi pareja no llama o no aparece a la hora acordada, me preocupa pen- &, 55 &, 31 \\
sar que está enojada/o conmigo. & & \\
7. Cuando mi pareja debe ausentarse por algunos días, me siento ansioso/a. &, 65 &, 43 \\
8. Cuando discuto con mi pareja me preocupa que deje de quererme. &, 65 &, 42 \\
9. He amenazado con hacerme daño para que mi pareja no me deje. &, 64 &, 41 \\
10. Me considero una persona débil. &, 64 &, 29 \\
11. Necesito mucho que mi pareja me exprese afecto. &, 65 &, 42 \\
12. Necesito tener a una persona que me considere especial. &, 42 &, 18 \\
13. Cuando tengo una discusión con mi pareja me siento vacío/a. &, 61 &, 37 \\
14 Me siento muy mal, si mi pareja no me expresa frecuentemente afecto. &, 65 &, 42 \\
15. Siento temor a que mi pareja me abandone. &, 77 &, 60 \\
16. Si mi pareja me propone una actividad dejo todo para estar con él/ella. &, 59 &, 35 \\
17. Me siento intranquilo cuando no conozco donde está mi pareja. &, 65 &, 42 \\
18. Siento una fuerte sensación de vacío cuando estoy solo/a. &, 69 &, 49 \\
19. No me agrada la soledad. &, 44 &, 18 \\
20. Soy capaz de hacer cosas temerarias, hasta arriesgar mi vida, por conser- &, 67 &, 45 \\
var el amor de mi pareja. & 38,78 \\
21. Si tengo planes y mi pareja aparece, los cambio solo por estar con él/ella. &, 58 &, 34 \\
22. Me alejo demasiado de mis amigos cuando tengo una relación de pareja. &, 58 &, 34 \\
23. Me divierto solamente cuando estoy con mi pareja. &, 58 &, 34 \\
Carga/Comunalidad media &, 65 &, 38 \\
\hline Autovalor (cantidad de varianza total del factor) & & 82 \\
Porcentaje de varianza explicada por el factor & & \\
\hline
\end{tabular}

Nota: $\mathrm{FG}=$ dependencia emocional (un solo factor); $\mathrm{h}^{2}=$ comunalidad antes de la rotación factor general. 
La tabla 2 presenta el análisis factorial exploratorio con un factor. El análisis de un factor explica el 38,78\% de la varianza del constructo dependencia emocional. En esta misma tabla, se aprecia que las cargas medias en el análisis de un factor son de ,65. Por otro lado, la comunalidad media es de ,38. En relación a los autovalores, el análisis de un factor presenta un valor de 8,92 .

Análisis factorial confirmatorio. El AFC se utilizó para verificar la estructura interna de la escala. Para ello, se compararon seis modelos: modelo 1 , con un solo factor; modelo 2, con seis factores relacionados; modelo 3 , con seis factores independientes; modelo 4, con seis factores específicos con un factor general; modelo 5 , con seis factores con un factor de segundo orden; y el modelo 6 , con un único factor con seis factores ortogonales. Los modelos 4 y 6 pueden ser considerados modelos bifactor.
Los resultados se presentan en la tabla 3 , indicando que el modelo 6 presenta las mejores bondades de ajuste: (S-B $\chi^{2}$ (201) $=223,04 ; p<, 05 ; \mathrm{S}-\mathrm{B} \chi^{2} / g l=1,10 ; \mathrm{CFI}=$ ,99; NNFI = ,98; SRMR = ,06; RMSEA = ,02; $\mathrm{AIC}=-178,76)$.

En vista de que el modelo 6 es bifactor, se utilizaron los índices de resistencia (Ríos \& Wells, 2014), evidenciando que el ECV fue de ,74 y el PUC, de ,83; con estos valores se indica una tendencia a la unidimensionalidad, tal como el AFE evidenció.

Fiabilidad. En la tabla 4 se presentan las cargas factoriales para cada uno de los modelos. Se observa que, en promedio, las cargas factoriales del modelo $3\left(\lambda_{\text {promedio }}=\right.$ $, 64)$, son mayores que el resto. Le sigue el modelo 2 y 5 , con el mismo valor $\left(\lambda_{\text {promedio }}\right.$ $=, 63)$, y $6\left(\lambda_{\text {promedio }}=, 51 /, 35\right)$. El modelo 4 presentó, en promedio, las cargas factoriales más bajas $\left(\lambda_{\text {promedio }}=, 35 /, 51\right)$.

Tabla 3

Índices de ajuste estadístico de dos modelos del CDE ( $\mathrm{N}=320)$

\begin{tabular}{|c|c|c|c|c|c|c|c|}
\hline Modelo & $\begin{array}{l}\text { S-B } \chi^{2} \\
(\mathrm{gl})\end{array}$ & S-B $\chi^{2} / g 1$ & SRMSR & CFI & NNFI & $\begin{array}{l}\text { RMSEA } \\
\text { [IC 90\%] }\end{array}$ & AIC \\
\hline 1 & $\begin{array}{c}603,41 \\
(230)\end{array}$ & 2,62 & ,08 & ,78 & ,76 & $\begin{array}{c}, 07 \\
{[, 06 ;, 08]}\end{array}$ & 143,40 \\
\hline 2 & $\begin{array}{c}379,16 \\
(215)\end{array}$ & 1,76 & ,06 & ,88 & ,86 & $\begin{array}{c}, 06 \\
{[, 06 ;, 07]}\end{array}$ & 69,73 \\
\hline 3 & $\begin{array}{c}926,87 \\
(230)\end{array}$ & 4,03 &, 24 &, 59 &, 55 & $\begin{array}{c}, 10 \\
{[, 09 ;, 10]}\end{array}$ & 466,87 \\
\hline 4 & $\begin{array}{c}376,33 \\
(206)\end{array}$ & 1,82 & ,06 & ,90 & ,88 & $\begin{array}{c}, 05 \\
{[, 04 ;, 06]}\end{array}$ & $-35,66$ \\
\hline 5 & $\begin{array}{c}406,63 \\
(223)\end{array}$ & 1,82 & ,06 & ,89 & ,88 & $\begin{array}{c}, 05 \\
{[, 04 ;, 06]}\end{array}$ & $-39,36$ \\
\hline 6 & $\begin{array}{c}223,04 \\
(201)\end{array}$ & 1,10 & ,06 & ,99 & ,98 & $\begin{array}{c}, 02 \\
{[, 00 ;, 03]}\end{array}$ & $-178,96$ \\
\hline
\end{tabular}

Nota: $\mathrm{S}-\mathrm{B}=$ método de estimación robusta de Satorra-Bentler; $\mathrm{gl}=$ grados de libertad; SRMSR = raíz residual estandarizada cuadrática media; CFI = índice de ajuste comparativo; NNFI = índice de ajuste no normado; RMSEA = error cuadrático medio de aproximación; AIC = criterio de información de Akaike. 
Asimismo, se calculó el omega (tabla 4). El mayor coeficiente lo presentó el modelo $3(\omega=, 93)$; el modelo 2 y 5 tiene un igual coeficiente $(\omega=, 92)$, seguido del modelo $6(\omega=, 90)$ y $1(\omega=, 88)$; mientras que el modelo 4 es el más bajo de todos $(\omega=, 85)$.
En cuanto a los valores de la AVE, el valor más próximo a ,50 (Fornell \& Larcker, 1981) lo obtuvo el modelo 2 y 3 (AVE = ,36), seguido del modelo $5(\mathrm{AVE}=, 35)$, el modelo $6(\mathrm{AVE}=, 27)$ y el modelo 1 $(\mathrm{AVE}=, 26)$, mientras que el valor más bajo lo presentó el modelo $4(\mathrm{AVE}=, 16)$.

Tabla 4

Saturaciones de los ítems en los diversos modelos $(\mathrm{N}=320)$

\begin{tabular}{|c|c|c|c|c|c|c|}
\hline Items & Modelo 1 & Modelo 2 & Modelo 3 & Modelo 4 & Modelo 5 & Modelo 6 \\
\hline 1 &, 32 &, 37 &, 41 &, $27 /, 33$ &, 37 & ,33/,27 \\
\hline 2 & 60 & 61 & ,64 & ,21/,58 & ,62 &, $58 /, 21$ \\
\hline 3 & ,31 & ,66 & 1 &, $65 /, 28$ & ,61 & ,28/,44 \\
\hline 4 & ,40 &, 77 &, 51 &, $63 /, 38$ & ,84 & ,38/,93 \\
\hline 5 & ,49 & 62 &, 62 & ,48/,43 &, 58 &, $43 /, 48$ \\
\hline 6 & 62 & ,64 & ,64 & ,27/,59 & ,64 &, $59 /, 27$ \\
\hline 7 & 63 & 65 & ,68 & ,41/,58 & ,65 & ,58/,41 \\
\hline 8 & 66 & 69 &, 71 & ,49/,62 & ,69 & ,62/,38 \\
\hline 9 & ,45 &, 55 &, 58 & ,38/,45 &, 55 &, $45 /, 38$ \\
\hline 10 &, 56 & ,66 &, 65 & ,30/,58 & ,66 &, $58 /, 30$ \\
\hline 11 & ,64 &, 72 &, 76 & ,42/,62 &, 73 &, $62 /, 41$ \\
\hline 12 &, 38 & ,38 &, 36 &, $11 /, 37$ &, 38 & ,37/,11 \\
\hline 13 &, 72 &, 73 & ,69 & ,09/,73 &, 73 & ,73/,09 \\
\hline 14 & ,65 &, 72 &, 70 & ,31/,64 &, 74 &, $64 /, 31$ \\
\hline 15 &, 73 &, 75 &, 75 &, $15 /, 73$ &, 75 &, $74 /, 15$ \\
\hline 16 & ,49 & 65 & ,63 & ,45/,46 &, 65 &, $46 /, 45$ \\
\hline 17 &, 54 &, 54 &, 52 & , 10/,53 &, 54 &, $53 /, 10$ \\
\hline 18 & 63 &, 73 & ,67 &, $23 / 65$ & ,73 &, $65 /, 23$ \\
\hline 19 & ,46 &, 51 &, 56 & ,26/,48 &, 53 &, $48 /, 26$ \\
\hline 20 &, 53 & 61 &, 60 & ,27/,54 & ,61 &, $54 /, 27$ \\
\hline 21 & ,44 &, 73 &, 80 & ,74/,40 & ,74 &, $40 /, 74$ \\
\hline 22 & ,43 & ,65 & ,64 & ,47/,40 &, 65 &, $40 /, 47$ \\
\hline 23 & ,46 & ,59 &, 52 & ,30/,46 &, 57 & ,46/,30 \\
\hline$\lambda_{\text {promedio }}$ &, 53 & 63 & ,64 & ,35/,51 & ,63 &, $51 /, 35$ \\
\hline$\omega$ & ,88 & ,92 & ,93 &, 85 & ,92 & ,90 \\
\hline AVE & ,26 & ,36 &, 36 &, 16 &, 35 &, 27 \\
\hline $\mathrm{ECV}$ & & & & ,39 & &, 74 \\
\hline PUC & & & &, 83 & &, 83 \\
\hline
\end{tabular}

Nota $: \lambda_{\text {promedio }}=$ carga factorial promedio; $/=$ simboliza las cargas factorial del modelo bifactor; $\omega=$ coeficiente omega; $\mathrm{AVE}=$ varianza extraída promedio; $\mathrm{ECV}=$ varianza común explicada; $\mathrm{PUC}=$ porcentaje de correlaciones no contaminadas. 


\section{Discusión}

El objetivo principal del presente estudio, fue analizar las propiedades psicométricas de confiabilidad y validez del Cuestionario de Dependencia Emocional, de Lemos Hoyos y Londoño Arredondo (2006), en estudiantes universitarios. En primer lugar, los resultados del análisis descriptivo de los ítems evidenciaron que los ítems 9 (He amenazado con hacerme daño para que mi pareja no me deje), 10 (Me considero una persona débil) y 20 (Soy capaz de hacer cosas temerarias, hasta arriesgar mi vida, por conservar el amor de mi pareja) que conforman el factor Expresión limite en la escala original, presentaron una asimetría y curtosis mayor a $+/-1,5$ (Ferrando \& Anguiano-Carrasco, 2010; Pérez \& Medrano, 2010). Aquello está relacionado con la alta frecuencia de la alternativa 1 (Completamente falso de mî) que, en respuesta de las personas, no representan una conducta usual en ellos.

Es interesante observar que las autoras del CDE incluyen los ítems 9,10 y 20 en su cuestionario como parte de la dependencia emocional. Este suceso podría sugerir que la dependencia emocional se manifiesta de forma diferente en los estudiantes peruanos, en comparación con los colombianos, lo cual podría estar influido por la cultura (Fernández et al., 2010).

El análisis de la validez se realizó en dos fases. En primer lugar, se llevó a cabo el AFE. Para la determinación del número de factores, se utilizó el método de análisis paralelo, basado en el análisis factorial de rango mínimo (Timmerman \& Lorenzo-Seva, 2011), debido a que es el más preciso para establecer la estimación de la dimensionalidad de las variables (Escurra Mayaute \& Salas Blas, 2014; Ledesma \& Valero-Mora, 2007) y ser el más recomendado por políticas editoriales (Thom- pson \& Daniel, 1996). Se encontró, por medio de este método, que los ítems eran explicados por un solo factor. Este único factor explica cerca del $38,78 \%$ de la varianza, tomándose como criterio de la pertenencia de un ítem a un factor que las cargas factoriales sean iguales o mayores a ,30 (Kline, 1993; Nunnally, 1987).

En segundo lugar, debido a la diferencia teórica que existió en el AFE, que reportó que un solo factor subyace a todos los ítems, se tuvo que utilizar el AFC para verificar y comparar estos hallazgos con el modelo original, método que es considerado una aproximación fuerte a la validación de constructo (Messick, 1995; Pérez-Gil, Chacón Moscoso, \& Moreno Rodríguez, 2000).

El AFC indicó que el modelo 6 presenta valores de bondad de ajuste adecuados, si se compara con los demás modelos. En el presente estudio se utilizó la corrección para datos no-normales, método de estimación robusta de Satorra-Bentler (Satorra $\&$ Bentler, 2001), considerando dividir el $\mathrm{S}-\mathrm{B}$ y $\chi^{2}\left(\mathrm{~S}-\mathrm{B} / \chi^{2}\right)$, obteniendo un valor de 1,10; siendo aceptable al ser inferior a 4 (Byrne, 1989; Carmines \& McIver, 1981).

Se estimó, así mismo, el índice de ajuste no normado (NNFI $=, 98)$ y el índice de ajuste comparativo $(\mathrm{CFI}=, 99)$, los valores superiores a, 90 son considerados adecuados (MacCallum \& Austin, 2000). El CFI es importante para comparar, de forma general, el modelo estimado con un modelo nulo; que indica independencia entre las variables estudiadas (Hair et al., 2004; Manzano \& Zamora, 2010). Adicionalmente, se analizó el error cuadrático medio de aproximación (RMSEA $=, 02)$ y la raíz residual estandarizada cuadrática media $($ SRMR $=, 06)$, la cual resultó aceptable, al obtener un valor inferior a ,08 (Browne \& Cudeck, 1993). Estos dos 
últimos valores son significativos, al estar muy próximos a cero (Ferrando \& Anguiano-Carrasco, 2010; Formiga et al., 2011; Sánchez \& Sánchez, 1998). Finalmente, se estimó el criterio de información de Akaike (AIC = -178,96), siendo el valor más pequeño (Hancock \& Mueller, 2006). Con el fin de determinar si el CDE es multidimensional o unidimensional, se utilizaron los índices de resistencia que sugirieron que el comportamiento de los ítems sugiere un factor general.

El AFC realizado ha logrado identificar que el modelo 6, de un factor general con seis factores específicos, tiene aceptables valores de bondad de ajuste, y cuenta con valores de fiabilidad buenos, al menos para la muestra en estudio, siendo su omega $(\omega=, 90)$ y con un AVE de ,27; valor inferior a lo recomendado, que es ,50 (Fornell \& Larcker, 1981). Cabe mencionar que la validez factorial brinda evidencia favorable acerca de las mediciones referidas al constructo que se mide $(\mathrm{Mu}-$ ñiz, 1998). Estos hallazgos son diferentes a los encontrados en la escala original, debido a que se utilizaron diferentes mé- todos de extracción y rotación, empleándose solamente el AFE, lo que sí se comprueba es que el comportamiento de los ítems es de un factor general, influido por seis factores específicos.

No obstante, entre las limitaciones del estudio se deben señalar el muestreo no probabilístico y la poca representatividad de la muestra. Aquello lleva a considerar las conclusiones del estudio como hipótesis previas para investigaciones posteriores; donde se realicen muestreos probabilísticos y se examine el comportamiento del cuestionario en una población más amplia y diferente a la aquí estudiada. Por lo tanto, es importante señalar que los resultados obtenidos en el presente estudio no son concluyentes, es necesario continuar las investigaciones para encontrar mayores evidencias acerca de la validez y confiabilidad del cuestionario; utilizándose, así, otros métodos de validez como los planteados por Campbell y Fiske (1955), acerca de la validación mediante el método convergente y divergente, utilizando la matriz multirasgomultimétodo.

\section{Referencias}

Aiquipa T., J. (2012). Diseño y validación del inventario de dependencia emocional - IDE. Revista de Investigación en Psicología, 15(1), 133-145. Recuperado de http://revistasinvestigacion.unmsm.edu.pe/index.php/psico/article/view/3673/2946

Aiquipa Tello, J. J. (2015). Dependencia emocional en mujeres víctimas de violencia de pareja. Revista de Psicología (Perú, Lima), 33(2), 412-437. Recuperado de http://www.scielo.org.pe/pdf/psico/v33n2/a07v33n2.pdf

Bartlett, M. S. (1950). Tests of significance in factor analysis. British Journal of Statistical Psychology, 3(2), 77-85. http://dx.doi.org/10.1111/j.2044-8317.1950.tb00285.x

Batista-Foguet, J. M., Coenders, G., \& Alonso, J. (2004). Análisis factorial confirmatorio. $\mathrm{Su}$ utilidad en la validación de cuestionarios relacionados con la salud. Medicina Clínica, 122(1), 21-27. Recuperado de https://is.gd/nSprZi 
Bireda, M. R. (1998). Independencia emocional: una guía para liberarse de los sentimientos adictivos. Barcelona, España: Obelisco.

Bornstein, R. F., Geiselman, K. J., Eisenhart, E. A., \& Languirand, M. A. (2002). Construct validity of the relationship profile test: Links with attachment, identity, relatedness, and affect. Assessment, 9(4), 373-381.

http://dx.doi.org/10.1177/1073191102238195

Bornstein, R. F., Geiselman, K. J., Gallagher, H. A., Ng, H. M., Hughes, E. E., \& Languirand, M. A. (2004). Construct validity of the relationship profile test: Impact of gender, gender role, and gender role stereotype. Journal of Personality Assessment, 82(1), 104-113. http://dx.doi.org/10.1207/s15327752jpa8201_15

Bornstein, R. F. \& O'Neill, R. M. (2000). Dependency and suicidality in psychiatric inpatients. Journal of Clinical Psychology, 56(4), 463-473.

http://dx.doi.org/10.1002/(SICI)1097-4679(200004)56:4<463::AID-JCLP2>3.0.CO;2-5

Browne, M. W. \& Cudeck, R. (1993). Alternative ways of assessing model fit. En K. A. Bollen \& J. S. Long (Eds.), Testing structural equation models (pp. 136-162). Newbury Park, California: SAGE.

Byrne, B. M. (1989). A primer of LISREL: Basic applications and programming for confirmatory factor analytic models. New York, New York: Springer-Verlag.

Campbell, D. T. \& Fiske, D. W. (1959). Convergent and discriminant validation by the multitrait-multimethod matrix. Psychological Bulletin, 56(2), 81-105. http://dx.doi.org/10.1037/h0046016

Carmines, E. G. \& Mclver, J. D. (1981). Analyzing models with unobserved variables: Analysis of covariance structures. En G. W. Bohinstedt \& E. F. Borgatta (Eds.), Social measurement: Current issues (pp. 65-115). Beverly Hills, California: SAGE

Castelló Blasco, J. (2005). Dependencia emocional: características y tratamiento. Madrid, España: Alianza Editorial.

Costello, A. B. \& Osborne, J. W. (2005). Best practices in exploratory factor analysis: Four recommendations for getting the most from your analysis. Practical Assessment, Research \& Evaluation, 10(7), 1-9. Recuperado de http://pareonline.net/getvn.asp?v=10\&n=7

Escurra Mayaute, M. \& Salas Blas, E. (2014). Construcción y validación del cuestionario de adicción a redes sociales (ARS). Liberabit, 20(1), 73-91. Recuperado de http://www.scielo.org.pe/pdf/liber/v20n1/a07v20n1.pdf

Fernández, A., Pérez, E., Alderete, A. M., Richaud, M. C., \& Fernández Liporace, M. (2010). ¿Construir o adaptar tests psicológicos? Diferentes respuestas a una cuestión controvertida. Evaluar, 10, 60-74. Recuperado de https://revistas.unc.edu.ar/index.php/revaluar/article/view/459/428

Ferrando, P. J. \& Anguiano-Carrasco, C. (2010). El análisis factorial como técnica de investigación en psicología. Papeles del Psicólogo, 31(1), 18-33. Recuperado de https://dialnet.unirioja.es/servlet/articulo?codigo $=3150810$ 
Formiga, N. S., Rique, J., Galvao, L., Camino, C., Mathias, A., \& Medeiros, F. (2011). Escala multidimensional de reatividade interpessoal - EMRI: Consistência estrutural da versão reduzida. Revista de Psicología (Trujillo, Perú), 13(2), 188-198. Recuperado de http://sisbib.unmsm.edu.pe/BVRevistas/rev_psicologia_cv/v13_2011_2/pdf/a05.pdf

Fornell, C. \& Larcker, D. F. (1981), Evaluating structural equations models with unobservable variables and measurement error. Journal of Marketing Research, 18(1), 39-50. http://dx.doi.org/10.2307/3151312

Freiberg Hoffmann, A., Stover, J. B., de la Iglesia, G., \& Fernández Liporace, M. (2013). Correlaciones policóricas y tetracóricas en estudios factoriales exploratorios y confirmatorios. Ciencias Psicológicas, 7(2), 151-164. Recuperado de https://is.gd/tZrmhi

González-Jiménez, A. J. \& Hernández-Romera, M. del M. (2014). Emotional dependency based on the gender of young adolescents in Almeria, Spain. Procedia - Social and Behavioral Sciences, 132, 527-532.

http://dx.doi.org/10.1016/j.sbspro.2014.04.348

Hair, J. F., Anderson, R. E., Tatham, R. L., \& Black, W. C. (2004). Análisis multivariante. Madrid, España: Prentice Hall.

Hancock, G. R. \& Mueller, R. O. (Eds.). (2006). Structural equation modeling: A second course. Greenwich, Connecticut: Information Age Publishing.

Harrington, D. (2008). Confirmatory factor analysis. New York, New York: Oxford University Press.

http://dx.doi.org/10.1093/acprof:oso/9780195339888.001.0001

Hernández Sampieri, R., Fernández Collado, C., \& Baptista Lucio, P. (2014). Metodología de la Investigación. Distrito Federal, México: McGraw-Hill.

Hirschfeld, R. M. A., Klerman, G. L., Gouch, H. G., Barrett, J., Korchin, S. J., \& Chodoff, P. (1977). A measure of interpersonal dependency. Journal of Personality Assessment, 41(6), 610-618. http://dx.doi.org/10.1207/s15327752jpa4106_6

Jaller Jaramillo, C. \& Lemos Hoyos, M. (2009). Esquemas desadaptativos tempranos en estudiantes universitarios con dependencia emocional. Acta Colombiana de Psicología, 12(2), 77-83. Recuperado de http://www.scielo.org.co/pdf/acp/v12n2/v12n2a08.pdf

Kaiser, H. F. (1970). A second generation little jiffy. Psychometrika, 35(4), 401-415. http://dx.doi.org/10.1007/BF02291817

Kaiser, H. F. (1974). An index of factorial simplicity. Psychometrika, 39(1), 31-36. http://dx.doi.org/10.1007/BF02291575

Kline, P. (1986). A handbook of test construction: Introduction to psychometric design. London, England: Methuen.

Kline, P. (1993). An easy guide to factor analysis. Newbury Park, California: SAGE. http://dx.doi.org/10.4324/9781315788135 
Kline, R. B. (2011). Principles and practice of structural equation modeling. New York, New York: Guilford Press.

Ledesma, R. D. \& Valero-Mora, P. (2007). Determining the number of factors to retain in EFA: An easy-to-use computer program for carrying out parallel analysis. Practical Assessment, Research \& Evaluation, 12(2), 1-11. Recuperado de http://citeseerx.ist.psu.edu/viewdoc/download?doi=10.1.1.371.9140\&rep=rep1\&type=pdf

Lemos Hoyos, M., Jaller Jaramillo, C., González Calle, A. M., Díaz León, Z. T., \& De la Ossa, D. (2012). Perfil cognitivo de la dependencia emocional en estudiantes universitarios en Medellín, Colombia. Universitas Psychologica, 11(2), 395-404. Recuperado de http://www.scielo.org.co/pdf/rups/v11n2/v11n2a04.pdf

Lemos Hoyos, M. \& Londoño Arredondo, N. H. (2006). Construcción y validación del cuestionario de dependencia emocional en población colombiana. Acta Colombiana de Psicología, 9(2), 127-140. Recuperado de http://www.scielo.org.co/pdf/acp/v9n2/v9n2a12.pdf

Lemos Hoyos, M., Londoño Arredondo, N. H., \& Zapata Echavarría, J. A. (2007). Distorsiones cognitivas en personas con dependencia emocional. Informes Psicológicos, 9(9), 55-69. Recuperado de

https://revistas.upb.edu.co/index.php/informespsicologicos/article/view/1707/1650

Lorenzo-Seva, U. (1999). Promin: A method for oblique factor rotation. Multivariate Behavioral Research, 34(3), 347-356. http://dx.doi.org/10.1207/S15327906MBR3403_3

MacCallum, R. C. \& Austin, J. T. (2000). Applications of structural equation modeling in psychological research. Annual Review of Psychology, 51(1), 201-226. http://dx.doi.org/10.1146/annurev.psych.51.1.201

Manzano, A. \& Zamora, S. (2010). Sistema de ecuaciones estructurales: una herramienta de investigación. Distrito Federal, México: Centro Nacional de Evaluación para la Educación Superior.

McDonald, R. P. (1999). Test theory: A unified treatment. Mahwah, New Jersey: L. Erlbaum Associates. http://dx.doi.org/10.4324/9781410601087

Mellody, P. (2006). La adicción al amor. Barcelona, España: Obelisco.

Méndez Zavala, A. W., Favila Figueroa, M., A., Valencia Cruz, A., \& Díaz Loving, R. (2012). Adaptación y propiedades psicométricas del cuestionario de dependencia emocional pasiva en una muestra mexicana. Uaricha Revista de Psicología, 9(19), 113-128. Recuperado de http://revistauaricha.umich.mx/Articulos/uaricha_0919_113-128.pdf

Messick, S. (1995). Standards of validity and the validity of standards in performance assessment. Educational Measurement: Issues and Practice, 14(4), 5-8. http://dx.doi.org/10.1111/j.1745-3992.1995.tb00881.x

Montero, I. \& León, O. G. (2007). A guide for naming research studies in psychology. International Journal of Clinical and Health Psychology, 7(3), 847-862. Recuperado de http://www.aepc.es/ijchp/articulos_pdf/ijchp-256.pdf 
Morales Vallejo, P. (2013). El análisis factorial en la construcción e interpretación de tests, escalas y cuestionarios. Madrid, España: Universidad Pontificia Comillas.

Muñiz, J. (1998). Teoría clásica de los test. Madrid, España: Pirámide.

Muñiz, J. \& Hambleton, R. K. (1996). Directrices para la traducción y adaptación de los tests. Papeles del Psicólogo, 66, 63-70. Recuperado de http://www.papelesdelpsicologo.es/vernumero.asp?id=737

Norwood, R. (1985). Las mujeres que aman demasiado. Buenos Aires, Argentina: Javier Vergara Editor.

Nunnally, J. (1987). Teoría psicométrica. Distrito Federal, México: Trillas.

Pérez, E. R. \& Medrano, L. (2010). Análisis factorial exploratorio: bases conceptuales y metodológicas. Revista Argentina de Ciencias del Comportamiento, 2(1), 58-66. Recuperado de http://www.revistas.unc.edu.ar/index.php/racc/article/view/5270/5433

Pérez-Gil, J. A., Chacón Moscoso, S., \& Moreno Rodríguez, R. (2000). Validez de constructo: el uso de análisis factorial exploratorio-confirmatorio para obtener evidencias de validez. Psicothema, 12(2), 442-446. Recuperado de http://www.psicothema.com/pdf/601.pdf

R Development Core Team (2007). R: A language and environment for statistical computing. (3.1.2) [Software de computación en línea] Viena, Austria: R Foundation for Statistical Computing. Recuperado de http://cran.r-project.org/

Rathus, J. H. \& O'Leary, K. D. (1997). Spouse-Specific Dependency Scale: Scale development. Journal of Family Violence, 12(2), 159-168.

http://dx.doi.org/10.1023/A:1022884627567

Rios, J. \& Wells, C. (2014). Validity evidence based on internal structure. Psicothe$m a, 26(1), 108-116$.

http://dx.doi.org/10.7334/psicothema2013.260

Riso, W. (1999). ¿Amar o depender?: Cómo superar el apego afectivo y hacer del amor una experiencia plena y saludable. Bogotá, Colombia: Editorial Norma.

Sanathara, V. A., Gardner, C. O., Prescott, C. A., \& Kendler, K. S. (2003). Interpersonal dependence and major depression: Aetiological interrelationship and gender differences. Psychological Medicine, 33(5), 927-931. http://dx.doi.org/10.1017/s0033291703007542

Sánchez, E. \& Sánchez, M. (1998). Los modelos de estructuras de covarianza como método de validación de constructo. En V. Manzano \& M. Sánchez (Comps.), Investigación del comportamiento: innovaciones metodológicas y estrategias de docencia (pp. 101-112). Sevilla, España: Instituto Psicosociológico Andaluz de Investigaciones.

Satorra, A. \& Bentler, P. M. (2001). A scaled difference chi-square test statistic for moment structure analysis. Psychometrika, 66(4), 507-514.

http://dx.doi.org/10.1007/BF02296192 
Sirvent, C. \& Moral, M. V. (2005). Test de dependencias sentimentales TDS-100. Oviedo, España: Fundación Instituto Spiral.

Sirvent, C. \& Moral, M. V. (2007). La dependencia sentimental o afectiva. Anales de Psiquiatría, 23(3), 93-94.

Tabachnick, B. G. \& Fidell, L. S. (2007). Using multivariate statistics. Boston, Massachusetts: Pearson/Allyn \& Bacon.

Thompson, B. (2004). Exploratory and confirmatory factor analysis: Understanding concepts and applications. Washington, District of Columbia: American Psychological Association.

Thompson, B. \& Daniel, L. G. (1996). Factor analytic evidence for the construct validity of scores: A historical overview and some guidelines. Educational and Psychological Measurement, 56(2), 197-208.

http://dx.doi.org/10.1177/0013164496056002001

Timmerman, M. E. \& Lorenzo-Seva, U. (2011). Dimensionality assessment of ordered polytomous items with parallel analysis. Psychological Methods, 16(2), 209-220. http://dx.doi.org/10.1037/a0023353

Universitat Rovira i Virigili. (2013). Factor (9.20) [software de computación] Tarragona, España: Universitat Rovira i Virigili. Recuperado de https://is.gd/jsiekx 